\title{
Gravitational wave astronomy, relativity tests, and massive black holes
}

\author{
Peter L. Bender \\ (For the LISA International Science Team) \\ JILA, Univ. of Colorado and NIST
}

\begin{abstract}
The gravitational wave detectors that are operating now are looking for several kinds of gravitational wave signals at frequencies of tens of Hertz to kilohertz. One of these is mergers of roughly $10 M_{\odot} \mathrm{BH}$ binaries. Sometime between now and about 8 years from now, it is likely that signals of this kind will be observed. The result will be strong tests of the dynamical predictions of general relativity in the high field regime. However, observations at frequencies below $1 \mathrm{~Hz}$ will have to wait until the launch of the Laser Interferometer Space Antenna (LISA), hopefully only a few years later. LISA will have 3 main objectives, all involving massive BHs. The first is observations of mergers of pairs of intermediate mass $\left(100\right.$ to $\left.10^{5} M_{\odot}\right)$ and higher mass BHs at redshifts out to roughly $\mathrm{z}=10$. This will provide new information on the initial formation and growth of BHs such as those found in most galaxies, and the relation between BH growth and the evolution of galactic structure. The second objective is observations of roughly $10 M_{\odot}$ BHs, neutron stars, and white dwarfs spiraling into much more massive BHs in galactic nuclei. Such events will provide detailed information on the populations of such compact objects in the regions around galactic centers. And the third objective is the use of the first two types of observations for testing general relativity even more strongly than ground based detectors will. As an example, an extreme mass ratio event such as a $10 M_{\odot} \mathrm{BH}$ spiraling into a galactic center $\mathrm{BH}$ can give roughly $10^{5}$ observable cycles during about the last year before merger, with a mean relative velocity of $1 / 3$ to $1 / 2$ the speed of light, and the frequencies of periapsis precession and Lense-Thirring precession will be high. The LISA Pathfinder mission to prepare for LISA is scheduled for launch in 2011.
\end{abstract}

Keywords. Gravitational waves, massive black holes, structure formation, astrophysics, relativity tests

\section{Introduction}

A number of ground-based gravitational wave detectors based on laser interferometers are now in operation. This includes the following observatories: the LIGO observatories in Hanford, WA and Livingston, LA; the joint Italian-French VIRGO observatory near Pisa, Italy; the German-British GEO-600 observatory near Hannover, Germany; and the TAMA observatory in Mitaka, Japan. Major upgrades of the LIGO observatories over the next six years or so have been approved, and comparable improvements in sensitivity are expected at other sites. (See also preceeding article: [Schutz (2009b)].)

With the improvements in sensitivity that are planned, the predicted event rate for gravitational wave detections range from a few per month to tens of events per day. Most of the events are expected to be from mergers of roughly $10 \mathrm{M}_{\odot}$ black holes or of neutron stars. However, some may also be from supernova explosions. Despite the large uncertainty in the event rate, some black hole-black hole mergers are likely to have high enough signal-to-noise ratios (SNRs) so that their gravitational wave signals will provide strong tests of the predictions of general relativity in strong fields. 
In the future, gravitational wave observations also are expected from the Laser Interferometer Space Antenna (LISA) [Stebbins (2006); Sallusti et al. (2009)]. The design, launch and operation of LISA are being planned as a joint mission of the European Space Agency (ESA) and NASA. The scientific objectives of LISA include both astrophysical studies of several types and detailed testing of gravitational theory. The general design of the LISA mission and its scientific objectives will be described briefly in this article. In addition, the preparations for LISA through the LISA Pathfinder mission will be discussed.

\section{General Design of the LISA Mission}

In two-armed laser interferometer gravitational wave detectors, the quantity that is measured is essentially the difference of the light travel times between two arms. When gravitational waves are present, they change the light travel times differently for the two arms. LISA will have three arms, so observations can be made on two different polarizations of the gravitational waves simultaneously.

The LISA antenna consists of three spacecraft that form a nearly equilateral triangle with side lengths of about 5 million $\mathrm{km}$. The center of the triangle follows a circular path around the Sun, roughly 50 million $\mathrm{km}$ behind the Earth. Each spacecraft orbit has an eccentricity e of about 0.01 and an inclination $i$ equal to $\sqrt{3}$ times the eccentricity. The initial conditions for the orbits are arranged so that the triangle maintains an inclination of 60 degrees to the ecliptic as it goes around the Sun.

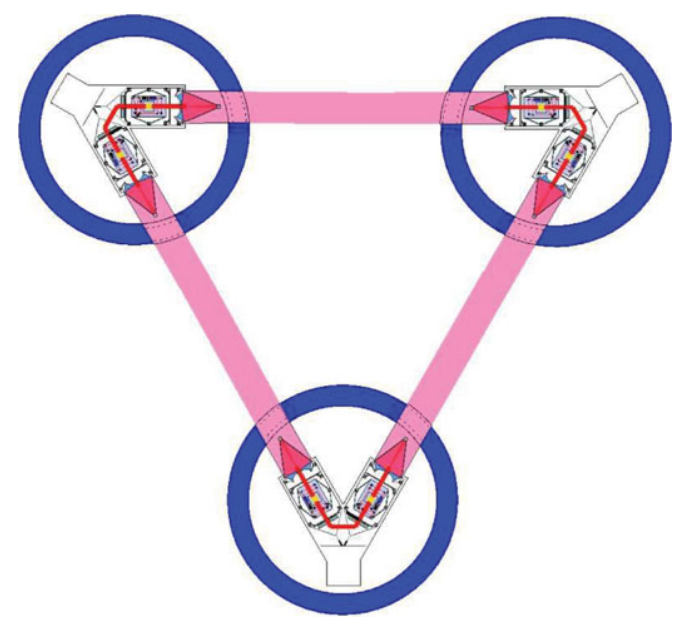

Figure 1. The LISA antenna.

Laser beams are sent both ways along each side of the triangle between the different spacecraft, as shown in Fig. 1. There are two optical assemblies in each spacecraft that transmit the laser beams through $40 \mathrm{~cm}$ diameter telescopes, and also receive the beams from the two distant spacecraft. The phase of the beat between the received beam and a portion of the transmitted beam is recorded as a function of time in each optical assembly. The resulting six records of the beat phase as a function of time are the main science output from the antenna, and are sent down to the ground every two days for analysis. The noise in the phase measurement over one second is equivalent to about 20 picometers in displacement, or $4 \times 10^{-21}$ in terms of the fractional change in arm length.

The other noise source that becomes important at frequencies below about 3 millihertz is spurious accelerations of the test masses that are used to correct for non-gravitational 
forces on the spacecraft [Carbone et al. (2007); Armando et al. (2009)]. Each optical assembly has a subsystem called a Gravitational Reference Sensor (GRS) mounted on the back of its telescope and optical bench, as shown in Fig. 2. At the center of each GRS is a test mass that is kept as free as possible of locally generated forces. Each test mass is a $4.6 \mathrm{~cm}$ cube of a gold-platinum alloy that has high density but low magnetic susceptibility. A cubical housing surrounds the test mass, with capacitive electrodes on its inside to sense displacements of the test mass with respect to the center of the cavity via ac bridge measurements. These measurements are used to servo-control the spacecraft to follow each test mass in its sensitive direction along the laser beam. Fairly weak electrical forces can also be applied via the capacitor plates to keep the test masses centered in their housings, and to avoid rotation of the test masses in their housings.

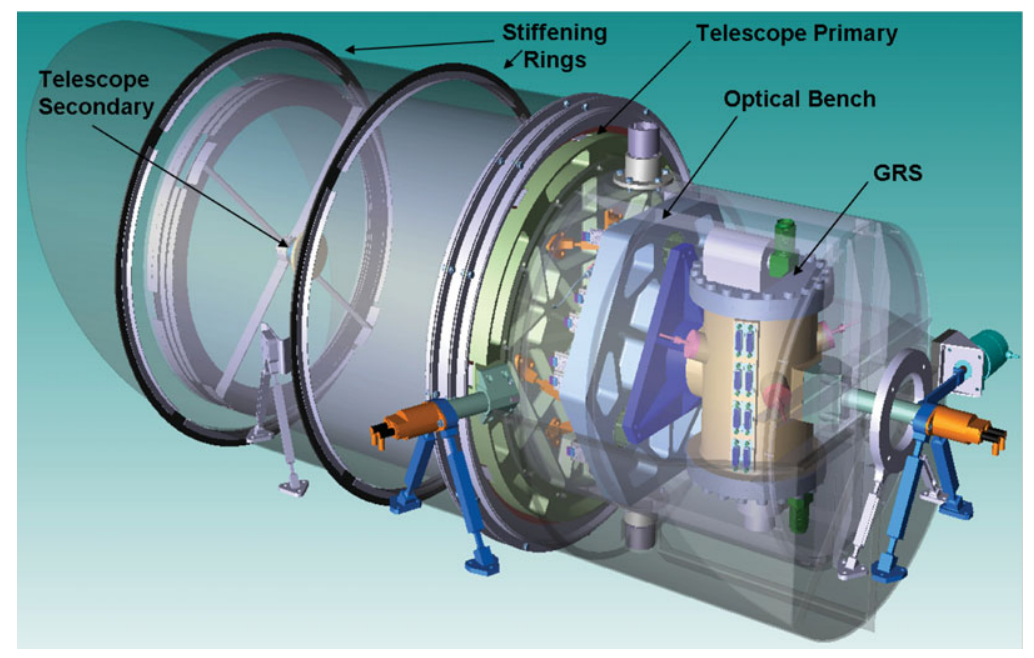

Figure 2. Optical assembly for LISA.

In contrast to previous missions, the gaps between the test masses and the housings around them are made quite large, about $4 \mathrm{~mm}$, in contrast to roughly $0.04 \mathrm{~mm}$ gaps in some previous missions. This greatly reduces possible spurious electrical forces due to work function differences between different surfaces. In addition, in order to minimize variations in forces acting on the test masses, the noise in the displacement of the housing with respect to each test mass is kept down to a level of 2 nanometers $/\left(\mathrm{Hz}^{0.5}\right)$ at frequencies down to 0.1 millihertz $(\mathrm{mHz})$. (This notation is to be interpreted as meaning that the mean square displacement noise power has to be less than $\left[\left(2 \times 10^{-9} \mathrm{~m}\right)^{2}\right] / \mathrm{Hz}$ down to $0.1 \mathrm{mHz}$.)

Such position-dependent perturbing forces could come from gravity gradients due to the mass distribution in the spacecraft, from work function differences between different surfaces, from magnetic field gradients, etc. An extensive error allocation budget for the various spurious acceleration sources is maintained, with a total level of $3 \times 10^{-15}$ $\mathrm{m} /\left(\mathrm{s}^{2}\right) /\left(\mathrm{Hz}^{0.5}\right)$ down to at least $0.1 \mathrm{mHz}$, including a $35 \%$ margin.

In ground-based gravitational wave detectors, the arms of the interferometer are kept equal to suppress noise due to laser frequency fluctuations. For LISA, since each spacecraft is in a nearly geodesic orbit around the Sun, it turns out that roughly $1 \%$ variations in the differences in the arm lengths cannot be avoided. However, these variations are essentially at annual, semi-annual and tri-annual periods, and thus are very smooth. 
The resulting Doppler shifts in the laser beams sent between the spacecraft can be as high as 15 Megahertz $(\mathrm{MHz})$, but highly accurate phase measurements as a function of time can still be made on the laser beat notes. This is done by specially designed phase meters that have been designed and thoroughly tested both in the US and in Europe [Shaddock et al. (2006); Wand et al. (2006)]. These phase measurements are made with respect to stable on-board oscillators, and the results are then sent down to the ground, where the data from the different spacecraft are combined and analyzed.

The threshold sensitivity curve for LISA is shown in Fig. 3 for the case of a monochromatic signal from a random direction that is observed for one year and that gives an amplitude signal-to-noise ratio (SNR) of 5 . The vertical axis is gravitational wave strain h. At frequencies above about $3 \mathrm{mHz}$ the curve is dominated by noise in measuring variations in the distance between the test masses at the ends of the arms, with comparable contributions from photon detection shot noise in the photo-detectors for the laser beat notes and from other possible sources of distance measurement noise. At lower frequencies the dominant noise source is the residual small spurious accelerations of the test masses.

\section{Strain Amplitudes During Last Year Before Coalescence}

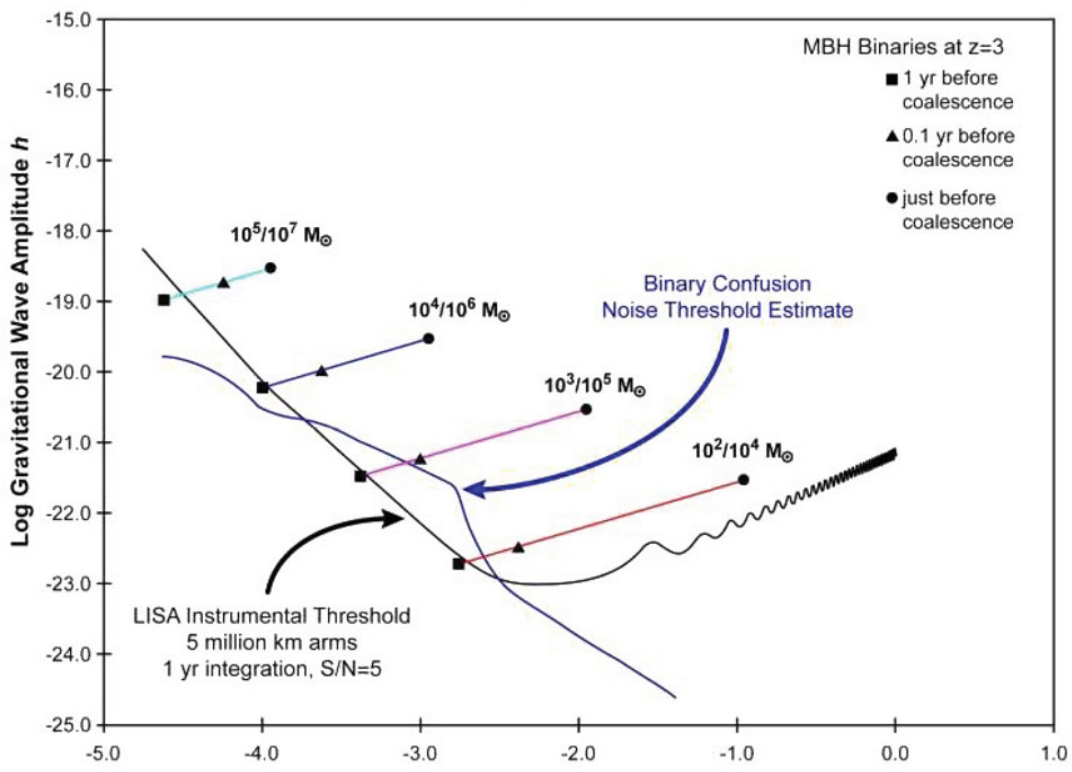

Figure 3. Threshold sensitivity curve and MBH binary signal strengths for LISA.

Also shown in Fig. 3 are the signal strengths that would be produced for 100:1 ratios of masses in massive black hole $(\mathrm{MBH})$ binaries merging at $\mathrm{z}=3$. The signal strength is shown at times of 1 year, 0.1 year, and 1 hour before reaching the last stable circular orbit. Since the threshold sensitivity curve is for 1 year of observations at a given frequency and the signal frequencies are changing rapidly, a separate calculation is needed to determine the integrated $\mathrm{S} / \mathrm{N}$ for the whole merger event. However, even the lowest mass merger shown would have an integrated $\mathrm{S} / \mathrm{N}>5$.

It should be noted that the threshold sensitivity curve shown at frequencies of 0.03 to $0.1 \mathrm{mHz}$ is based on the goal for the LISA mission, subject to verification by analysis. The additional curve shown is a rough estimate of the confusion noise threshold level 
likely to be present in the data because of unresolved signals from many close binary stars.

\section{Primary Scientific Objectives of LISA}

The first primary objective of the LISA mission, as discussed earlier, is the detection and detailed observation of gravitational wave signals from the mergers of intermediate mass and larger mass black holes throughout the universe. Current information indicates that most fairly large galaxies contain MBHs with masses of roughly $10^{6} \mathrm{M}_{\odot}$ or higher at their centers, and that the MBH mass depends quite directly on the mass of the central bulge and the dispersion of stellar velocities near the center. This indicates coevolution of the MBHs and the galaxies [Volonteri (2006); Di Matteo et al. (2008)]. When smaller pre-galactic structures merged, according to present theories of structure formation in the universe, the MBHs at their centers are likely to have spiraled down to the center of the resulting galaxy, formed a binary, and in most cases, merged. Thus observations of the signals from such mergers can provide valuable constraints on the history of structure formation and the growth of galaxies.

One constraint on our knowledge of this process is that we don't know how or when the intermediate mass black holes that later grew much larger in galactic nuclei were formed. One idea is that the stars that formed first in the universe had higher masses, and some of them experienced supernova explosions that left roughly $50 \mathrm{M}_{\odot}$ or larger black holes as remnants that later absorbed gas rapidly and grew [see e.g. Volonteri, Haardt, \& Madau (2003)]. Another is that mass segregation in dense star clusters may have led to such rapid accretion of a star at the center that normal stellar evolution was delayed [Freitag, Gürkan, \& Rasio (2006)]. A supernova explosion after an unusually high mass was reached could then result in an intermediate mass black hole, that later managed to get to the galactic center and grow. A third of the many suggested scenarios is that the very rapid accretion happened in a gas cloud of much higher mass and with very low metalicity, where stars hadn't yet formed. This would lead to the growth of an intermediate mass black hole within what is called a quasi-star. This is an object where the mass inflow rate is high enough to prevent normal stellar evolution for a quite long period. In this case, a black hole of perhaps $10^{3}$ or $10^{4} M_{\odot}$ could be formed before the rest of the surrounding mass was blown away by radiation [Begelman, Rossi, \& Armitage (2008)].

Various scenarios for when and how the intermediate mass black holes formed have been considered in what are called merger tree simulations of galaxy formation, including the mergers of pregalactic structures and the growth and mergers of the galactic center black holes in them, as a function of cosmic time (see e.g., [Sesana, Volonteri \& Haardt (2009)]). The resulting rates of events that LISA could detect are expected to be roughly tens to about 100 black hole merger events per year, depending on the intermediate mass black hole formation scenario that is assumed. The mass ratios tend to be roughly 10:1 or larger, and most events are expected at redshifts in the range of about 2 to 10 .

The second primary scientific objective for LISA is to observe the mergers of compact objects such as roughly $10 \mathrm{M}_{\odot}$ black holes, which are believed to be present in high numbers in galactic nuclei, with galactic center MBHs (see e.g., [Gair (2009)]). The rate of such events from which LISA could observe signals is again quite uncertain, but is estimated to be roughly tens to about 100 events per year. Such events would be observable out to redshifts of about $\mathrm{z}=1$, and are usually called Extreme Mass Ratio Inspirals, or EMRIs. Observing such events involving neutron stars and white dwarfs as well as black holes would provide new information on the conditions at the centers of 
galaxies containing MBHs, as well as on the scattering processes that lead to the compact objects being fed down to low angular momentum orbits around the black holes.

The third primary objective is closely related to the first two. It is to carry out detailed studies of whether the observed merger signals are in complete agreement with the predictions of general relativity (see e.g., [Schutz (2009a)]). For MBH binary mergers with comparable masses, advances in numerical relativity in the last few years indicate that accurate general relativistic templates for the signals will be available by the time they are needed, even for the merger and ringdown phases of the process. In some cases, the $\mathrm{S} / \mathrm{N}$ will be high, and the dynamics of the process will be tested, since both masses will be moving very rapidly.

For EMRIs, the tests will be even more stringent. For $10 \mathrm{M}_{\odot}$ and $10^{5}$ or $10^{6} \mathrm{M}_{\odot}$ as the black hole masses, there will be about 100,000 cycles of the signal that can be observed in the last year before merger. And even small deviations from the predictions of general relativity can be observed, as long as they don't have the same time signatures as the main parameters describing the masses and their spins. Because of the way that the 10 $\mathrm{M}_{\odot}$ black holes get scattered into orbits that can evolve mainly by gravitational wave energy loss, the initial orbits will be highly elliptical, and eccentricities of roughly 0.3 to 0.7 are expected right up to reaching the last stable orbit.

\section{Capture waveform: encoding the geometry}

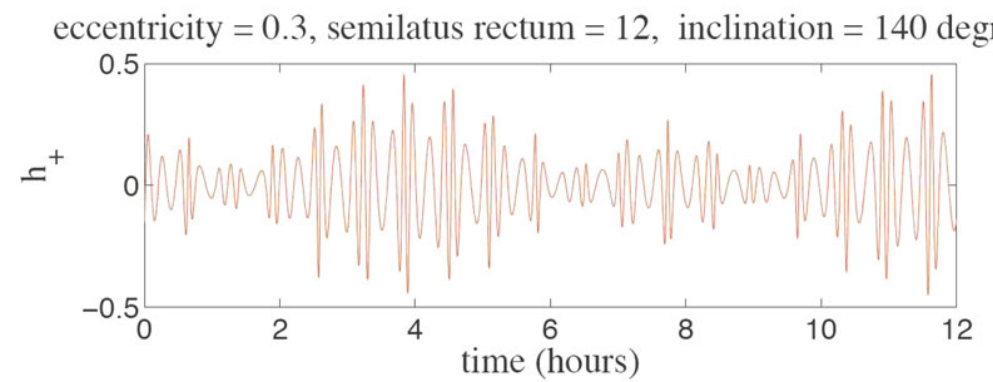

eccentricity $=0.7$, semilatus rectum $=6$, inclination $=60$ degrees

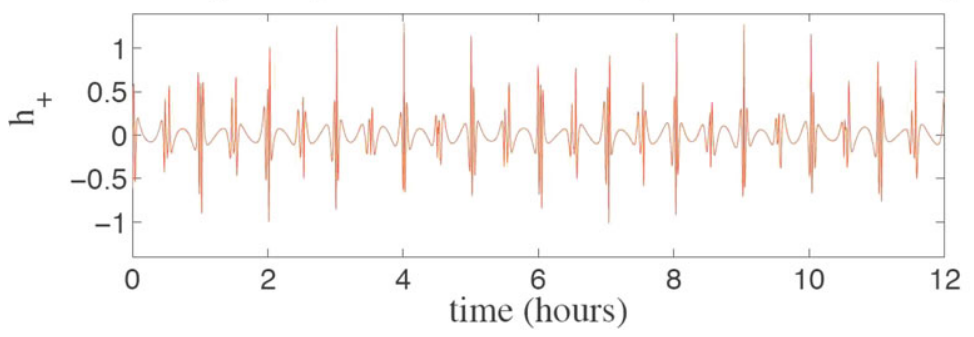

Figure 4. Examples of expected EMRI signals.

The speed at periapsis for $10 \mathrm{M}_{\odot}$ black hole EMRIs during the last year of inspiral is roughly $1 / 3$ or $1 / 2$ the speed of light, as mentioned earlier. The rate of precession of periapsis will be nearly as large as the radial motion frequency, and for rapidly spinning MBHs the Lense-Thirring precession can be comparable. Thus the observed signals will be complicated, involving perhaps $10^{4}$ cycles or more each of periapsis precession and Lense-Thirring precession during the last year. If there are even small errors in the predictions of general relativity, such as some small interaction between the above two types of precession or with the radial motion, it seems likely that this would show up in 
the EMRI results, but would have been missed in weak field or slow motion tests of the theory.

To illustrate the complexity of the relativistic effects discussed, two examples of possible signals as a function of time are shown in Fig. 4. This figure is from a White Paper [Schutz et al. (2009)] that was submitted in connection with the ASTRO2010 Decadal Survey to describe the scientific objectives for testing general relativity that can be carried out in the near future by a mission such as LISA. The possible tests are described in more detail there.

\section{LISA Pathfinder Mission}

The spurious acceleration level that is being planned on for the LISA mission is roughly four orders of magnitude better than has been aimed for or achieved on previous missions. Thus ESA decided in 2002 to provide a technology demonstration mission called LISA Pathfinder [McNamara (2006); McNamara et al. (2008); Racca \& McNamara (2009)] to validate the performance in space of the Gravitational Reference Sensors that were being developed to be flown on LISA [Armano et al. (2009)]. Initial design studies for the LISA GRSs were done at ONERA in Paris, but most of the later development of the GRSs for LISA Pathfinder and for LISA has been carried out at the University of Trento (see e.g., [Carbone et al. (2007)]).

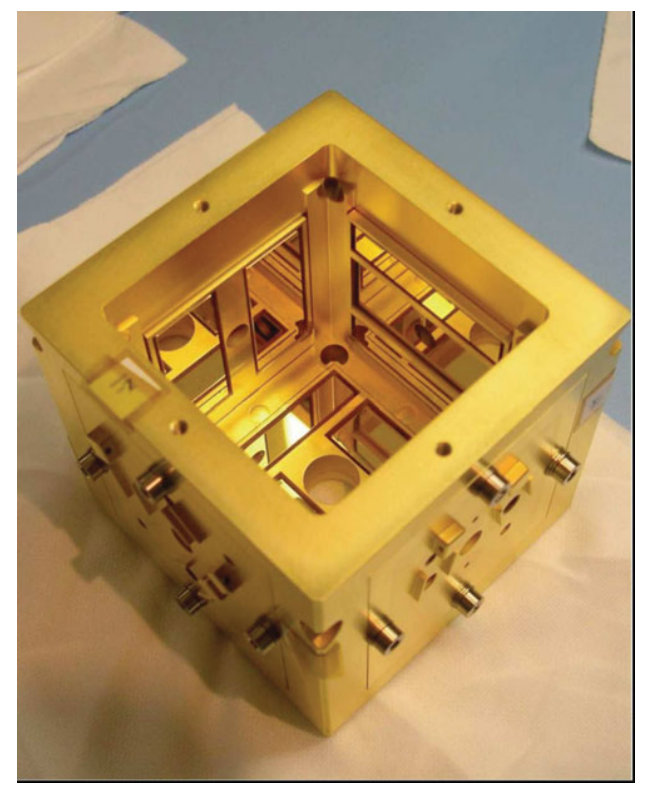

Figure 5. Electrode housing from Gravitational Reference Sensor designed for the LISA Pathfinder and LISA missions.

The electrode housing from one of the GRS units for LISA Pathfinder is shown in Fig. 5. The housing is made from molybdenum, which has good thermal conductivity. The electrodes mounted on its inner surface are thin sapphire plates with gold coatings on them. Holes in the housing permit a clamping mechanism to hold the test mass firmly in place against stops during launch, and then release it gently at the center of the housing when the spacecraft is in its final orbit. The test mass, housing, and clamping mechanism are contained in a vacuum enclosure, with sufficient getters to maintain a low pressure for more than the nominal mission lifetime of somewhat under a year. 
The basic LISA Pathfinder payload is shown in Fig. 6. It consists of two GRS units mounted on opposite ends of an optical bench made of low-expansion glass. Optical elements mounted on the optical bench form several interferometers that are used to monitor changes in the separations of the test masses in the GRS units with high precision. The GRS test mass spurious acceleration level is expected to be less than $3 \times 10^{-14}$ $\mathrm{m} / \mathrm{s}^{2} /\left(\mathrm{Hz}^{0.5}\right)$ at frequencies down to $1 \mathrm{mHz}$. A series of experimental investigations will be performed on LISA Pathfinder to validate the noise model that will permit extrapolation to the factor 10 better performance expected down to $0.1 \mathrm{mHz}$ for LISA because of the lower environmental disturbances that will be present on the LISA spacecraft.

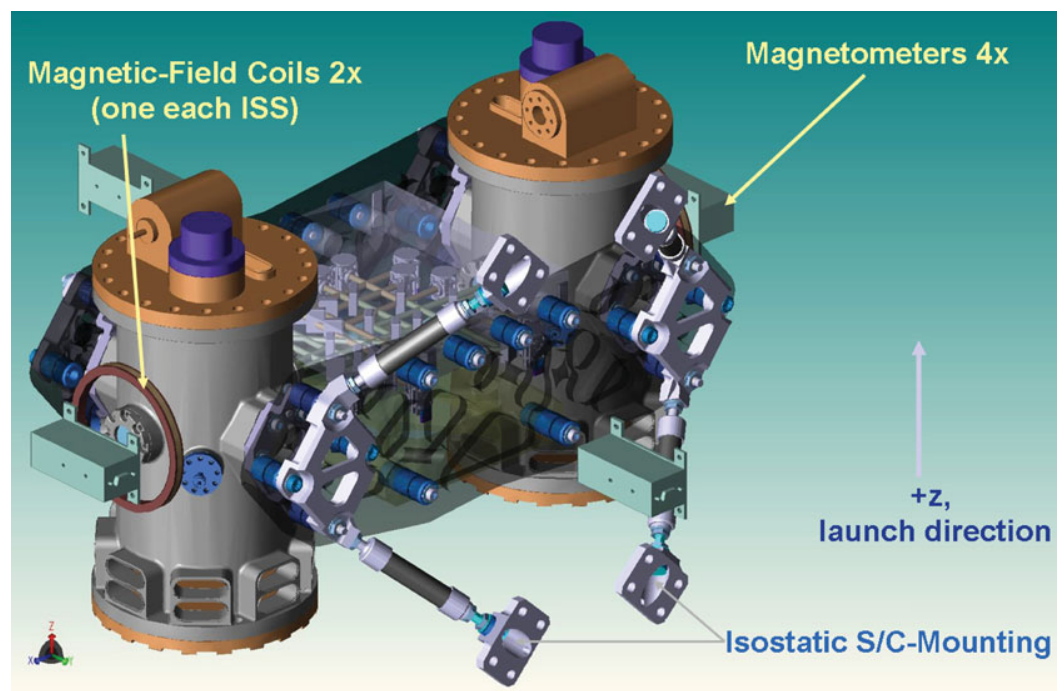

Figure 6. Basic science payload for the LISA Pathfinder mission.

In parallel with the development of the GRS for LISA Pathfinder, extensive laboratory tests have been done on a wide variety of possible spurious surface forces that could act on the test mass. These measurements have been carried out both at the University of Trento (see e.g., [Carbone et al. (2007)]) and at the University of Washington [Schlamminger et al. (2006); Pollack, Schlamminger, \& Gundlach (2006)]. Torsion pendulums supporting replicas of the test mass were used, and perturbing surfaces could be brought up close to the test mass to enhance the interactions. By operating near the thermal noise level for the torsion pendulums, limits on spurious accelerations from surface forces were brought down close to the goals set for LISA Pathfinder. However, when LISA Pathfinder is launched in 2011, the combination of space and ground tests of the GRS performance will provide even stronger evidence that no significant sources of spurious accelerations have been overlooked.

LISA Pathfinder also will provide tests of two types of micronewton thrusters that have been developed for use in LISA and in other missions. The main force acting on the LISA spacecraft will be roughly 30 micronewtons, due almost completely to solar radiation pressure. However, torques also will be present, so that rotations of the spacecraft as well as displacements with respect to one of the test masses inside will have to be controlled. Thus at least six small thrusters are needed, as well as an equal or larger number to provide sufficient redundancy. A similar number of micronewton thrusters will be flown on LISA Pathfinder. They will include both cesium ion thrusters developed under ESA 
support and thrusters that emit small but highly charged liquid droplets developed under NASA support.

\section{Conclusions}

The LISA mission is expected to provide unique new information on the development of structure in the universe, the origin and growth of intermediate mass and larger mass black holes, the co-evolution of galaxies and of the massive black holes at their centers, and the population of stellar mass compact objects around the galactic center black holes. In addition, it will provide nearly ideal tests of the predictions of general relativity from the gravitational wave signals observed during the mergers of different mass combinations of massive black holes. The LISA Pathfinder mission is scheduled for launch in 2011, to validate the performance of the new technology to be used on the LISA mission.

\section{References}

Armano, M. et al., 2009, Class $\mathcal{E}$ Quantum Grav. 26(9), 094001

Begelman, M. C., Rossi, E. M., \& Armitage, P. J., 2008, Mon. Not. R. Astron. Soc. 387, 1649

Carbone, L., et al., 2007, Phys. Rev. D 75(4), 042001

Di Matteo, T., et al., 2008, ApJ 676, 33

Freitag, M., Gürkan, M. A., \& Rasio, F. A., 2006, Mon. Not. R. Astron. Soc. 368, 141

Gair, J. R., 2009, Class \& Quantum Grav. 26(9), 094034

Hughes, S. A., 2006, in Laser Interferometer Space Antenna, AIP Conf. Proc. 873 (Melville, N. Y., Eds. S. M. Merkowitz and J. C. Livas), 13

McNamara, P. W., 2006, in Laser Interferometer Space Antenna, loc cit, 49

McNamara, P., Vitale, S., \& Danzmann, K., 2008, Class. \& Quantum Grav. 25(11), 114034

Pollack, S. E., Schlamminger, S., \& Gundlach, J. H., 2006, in Laser Interferometer Space Antenna, loc cit, 158

Racca, G. \& McNamara, P., 2009, Space Sci. Rev., (submitted)

Sallusti, M., et al., 2009, Class. \& Quantum Grav. 26(9), 094015

Schlamminger, S., et al., 2006, in Laser Interferometer Space Antenna, loc cit, 151

Schutz, B. F., 2009a, Class. \&3 Quantum Grav. 26(9), 094020

Schutz, B. F., 2009b, this proceedings, 234

Schutz, B. F., et al., 2009, "Will Einstein Have the Last Word on Gravity?" White Paper submitted to the ASTRO2010 Decadal Survey on Astronomy and Astrophysics

Sesana, A., Volonteri, M., \& Haardt, F., 2009, Class. \& Quantum Grav. 26(9), 094033

Shaddock, D., et al., 2006, in Laser Interferometer Space Antenna, loc cit, 654

Stebbins, R. T., 2006, in Laser Interferometer Space Antenna, loc cit, 3

Volonteri, M., 2006, in Laser Interferometer Space Antenna, loc cit, 61

Volonteri, M., Haardt, F., \& Madau, P., 2003, Astrophys. J. 582, 599

Wand, V., et al., 2006, in Laser Interferometer Space Antenna, loc cit, 689 